CASE RECORDS of the MASSACHUSETTS GENERAL HOSPITAL

Nancy Lee Harris, M.D., Editor Jo-Anne O. Shepard, M.D., Associate Editor

Sally H. Ebeling, Assistant Editor
Founded by Richard C. Cabot

Eric S. Rosenberg, M.D., Associate Editor Alice M. Cort, M.D., Associate Editor Christine C. Peters, Assistant Editor

\section{Fi:}

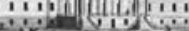

\title{
Case 15-2011: A 19-Year-Old South African Woman with Headache, Fatigue, and Vaginal Discharge
}

\author{
W.D. Francois Venter, F.C.P., Thumbi Ndung'u, B.V.M., Ph.D., \\ and Quarraisha Abdool Karim, Ph.D.
}

\begin{abstract}
From the Wits Reproductive Health and HIV Institute and the Department of Medicine, University of the Witwatersrand, Johannesburg (W.D.F.V.); the HIV Pathogenesis Programme (T.N.) and the Centre for the AIDS Programme of Research in South Africa (Q.A.K.), Doris Duke Medical Research Institute, Nelson R. Mandela School of Medicine, University of KwaZulu-Natal, Durban, South Africa; and the Department of Epidemiology, Mailman School of Public Health, Columbia University, New York (Q.A.K.).
\end{abstract}

N Engl J Med 2011;364:1956-64. Copyright (๑) 2011 Massachusetts Medical Society.
PRESENTATION OF CASE

Dr. Fundisiwe Chonco (Department of Virology, University of KwaZulu-Natal, Durban, South Africa): A 19-year-old woman was seen in a clinic at McCord Hospital in Durban, KwaZulu-Natal Province, South Africa (which is affiliated with Massachusetts General Hospital), because of headache, fatigue, and vaginal discharge.

The patient had been well until approximately 18 days earlier, when she began to have a vaginal discharge, followed by headache, fatigue, sore throat, and anorexia. On the fifth day of symptoms, she went to an outpatient clinic in an urban area. On examination, she had a vaginal discharge; the temperature and the remainder of the examination were reportedly normal. Two different rapid screening tests for human immunodeficiency virus type 1 (HIV-1) performed on a finger-stick blood sample were negative. Doxycycline, metronidazole, and ceftriaxone were administered according to South African guidelines for sexually transmitted diseases. The patient was invited to participate in a South African clinical research study; she accepted, and additional blood specimens were obtained. She was referred to the study clinic at McCord Hospital for follow-up, where she was seen 13 days later.

The patient reported persistent headache, fatigue, sore throat, and vaginal discharge. She was nulliparous and had a history of developmental delay after an accident during childhood; she was otherwise well. She was on no medications and had no known allergies. She was born in South Africa, lived with relatives in a house in an urban area of KwaZulu-Natal Province, and attended school. She had no history of sexually transmitted diseases and reported having had sexual intercourse four times with her 25-year-old boyfriend of 6 months, who had been her only partner. He reportedly had refused her request to use condoms.

On examination, the weight was $73 \mathrm{~kg}$, the temperature $36.0^{\circ} \mathrm{C}$, the blood pressure $93 / 59 \mathrm{~mm} \mathrm{Hg}$, and the pulse 82 beats per minute. There was a white, malodorous vaginal discharge; the remainder of the examination was normal except for findings consistent with the patient's chronic developmental delay. Additional blood specimens were obtained, and both these and specimens from the 
first clinic were sent to a reference laboratory for testing.

During the next 14 days, headache, fatigue, anorexia, sore throat, and vaginal discharge resolved, but a painful genital ulcer developed. The patient returned to the McCord Hospital clinic. She had no history of oral or genital ulcers and had not been sexually active since her visit to the first clinic. She was aware of HIV and that it is sexually transmitted.

On examination, the vital signs were normal. There was a painful pink ulcer, $5 \mathrm{~mm}$ by $10 \mathrm{~mm}$, on the labia majora on the left side, with undermined borders and without surrounding induration. There were palpable lymph nodes, $1 \mathrm{~cm}$ or smaller in diameter, in the submental, posterior cervical, and epitrochlear regions. The remainder of the examination was normal. Penicillin G benzathine and erythromycin were administered according to South African guidelines for genital ulcers. A rapid plasma reagin test was negative.

Diagnostic test results were reviewed.

DIFFERENTIAL DIAGNOSIS

Dr. W.D. Francois Venter: This young woman in KwaZulu-Natal, who is sexually active and not using condoms or contraceptives, has a vaginal discharge, followed by vague but relatively severe systemic symptoms, a painful genital ulcer, and lymphadenopathy. A travel history might add to an already long differential diagnosis, as would a history of medication use or visits to a traditional health worker, which commonly precede a visit to a nurse or doctor. Additional clinical details on the symptoms associated with the ulcer would allow for a more informed guess as to the cause.

The first question a local clinician would ask may seem startling to an outsider in view of this patient's relatively young age: Does this 19-yearold woman already have established HIV-1 infection? The patient's geographic and social demographic profile makes HIV infection a major issue to consider. A large study involving South African adolescents suggests that $13.8 \%$ of 19 -year-old women tested positive for HIV, and more than $30 \%$ tested positive by the age of 21 years. ${ }^{1}$ This extraordinary risk occurs even in the context of few sexual partners, making a more detailed sexual history unlikely to add much to this assessment. ${ }^{2}$ In addition, this patient has a neurologic deficit due to a childhood accident, which is also a risk factor for HIV infection. ${ }^{3}$

\section{SEXUALLY TRANSMITTED INFECTIONS}

The presence of a genital ulcer means that this patient has a high likelihood of HIV infection. In some South African provinces, more than $75 \%$ of patients who have genital ulcers and attend a primary care clinic are found to be HIV-positive. ${ }^{4}$ The cause of the ulcer may be predicted on the basis of local epidemiologic trends. ${ }^{5-7}$ Chancroid has almost disappeared from South Africa in the past two decades and syphilis rates have decreased to record low levels, whereas herpes has become steadily more prevalent and is now the most common cause of genital ulcers. ${ }^{5}$ Of patients with acute HIV infection, 5 to $15 \%$ have genital ulcers that are caused by HIV. ${ }^{8}$ This patient also has a vaginal discharge. In one recent study in South Africa, the two most common causes of vaginal discharge were bacterial vaginosis and trichomoniasis. ${ }^{9}$ As with genital ulcers, some studies show that women in this region of South Africa who presented with a vaginal discharge had a high risk of testing positive for HIV. ${ }^{1}$

Since clinical presentations of sexually transmitted infections correlate poorly with specific organisms, a syndromic therapeutic approach to a genital ulcer and discharge has been widely accepted and successful in South Africa. ${ }^{10,11}$ Specific tests that would help identify the organisms responsible for the discharge and ulcer include swabs and culture, serologic testing, and even biopsy. In reality, such an approach is rarely used in resource-poor countries.

\section{LYMPHADENOPATHY}

Could an illness other than a sexually transmitted infection comprehensively explain this patient's symptoms? Many illnesses can cause lymphadenopathy with systemic features, including other infectious diseases, connective-tissue diseases, sarcoid, neoplasms, and medications. There is an intimidating list of common infections in this region of South Africa, including hepatitis B, toxoplasmosis, schistosomiasis, streptococcus, rubella, Epstein-Barr virus, cat scratch disease, typhoid, infective endocarditis, bubonic plague, brucella, leptospirosis, abscesses, and typhus. Geography tends to limit other considerations such as Lyme disease, visceral leishmaniasis (kala-azar), try- 
panosomiasis, histoplasmosis, and other fungal infections.

\section{ACUTE HIV INFECTION}

Could this patient have acute HIV infection? This diagnosis would fit, since she is at high risk for newly acquired HIV infection, her antibody tests are negative, and she has a syndrome that is consistent with acute infection. The acute retroviral syndrome occurs 2 to 3 weeks after infection, usually coinciding with the onset of viremia, and is accompanied by several nonspecific symptoms, including fatigue, pharyngitis, weight loss, myalgias, and headache. ${ }^{12,13} \mathrm{An}$ astute clinician could rapidly limit the differential diagnosis with the use of available tools, including a good history taking and examination. Ruling out acute HIV infection should be the first step. Testing for HIV-1 RNA (viral load), testing for p24 antigen or HIV antibodies with the use of enzyme immunoassay (EIA), or simply repeating antibody testing over a period of a few weeks should establish a diagnosis of HIV infection and allow for further management steps.

DR. FRANCOIS VENTER'S DIAGNOSIS

Acute HIV infection with a concurrent ulcerative sexually transmitted infection.

\section{PATHOLOGICAL DISCUSSION}

Dr. Thumbi Ndung'u: The diagnostic test in this case was testing for HIV-1 RNA in a specimen of blood. Accurate diagnosis of HIV-1 infection forms the bedrock of many prevention, care, and clinical management strategies, and the World Health Organization has guidelines for expanded HIV testing globally. ${ }^{14}$ HIV-1 infection can be confirmed by testing of body fluids for the virus or for antiHIV antibodies.

The diagnostic hallmarks of acute HIV infection are a negative test for HIV-1 antibodies on EIA, an indeterminate or negative test for HIV-1 on Western blot analysis, and detection of HIV-1 RNA in plasma. In the outpatient setting, two rapid assays performed in parallel may be used in place of a third- or fourth-generation HIV EIA. In this case, the SD Bioline and Sensa Tri-Line HIV assays were used. Early detection of acute HIV infection is important because patients in the early phase are highly infectious, owing to the extreme- ly high viral loads in body fluids before the development of antiviral immune responses.

This patient was tested for HIV-1 infection at the first clinic with the use of rapid tests on whole blood. Rapid HIV tests are performed in the clinic or in a nearby laboratory, and results are usually available within 30 minutes. Although the results of a positive test must be confirmed with supplemental tests, rapid testing allows the clinician to screen for the presence of HIV-1 antibodies and implement a care plan before the patient leaves the clinic. The three methods of rapid testing that are commonly used are immunoconcentration, immunochromatography, and particle agglutination. In general, rapid tests use various HIV-1 antigens, typically located in the Gag and Env regions of HIV, that are fixed or coated on a test strip. If a specimen contains HIV antibodies, the antibodies will bind to the test antigens and trigger the detection system to generate a positive result. Typically, two different tests using different HIV antigens should be performed in parallel to make the presumptive diagnosis of HIV-1 infection. In this case, two rapid tests were performed on the initial specimen that was obtained at the first clinic and were negative. The patient was counseled and, after she agreed to participate in a research study (South African study EO36/06, Characterization of the Evolution of Adaptive Immune Responses in Acute HIV Clade C Virus Infection), blood was drawn and sent to the study laboratory for additional HIV testing. Testing for HIV antibodies with the use of EIA and HIV-1 Western blot was negative.

The plasma was then screened for acute HIV infection with the use of pooled nucleic acid testing. Pooling samples is a cost-effective screening strategy for the detection of the presence of HIV-1 RNA. In our clinic, where the prevalence of HIV infection is high, plasma samples from six persons are combined and then subjected to nucleic acid testing for the presence of HIV-1 RNA. The pool containing the sample from our patient was positive for HIV-1 RNA, prompting the individual testing of the constituent samples. The specimen from this patient revealed the presence of HIV-1 RNA (viral load, 12,445 copies per milliliter of plasma). HIV-1 RNA was not detected in any of the other samples included in the pool, confirming that the positive result was due to HIV-1 from this patient.

Our patient had several negative HIV-1 screen- 


\begin{tabular}{|c|c|c|c|c|c|c|}
\hline \multirow[t]{2}{*}{ Variable } & \multirow{2}{*}{$\begin{array}{l}\text { First Clinic } \\
13 \text { Days before } \\
\text { Presentation } \\
\text { at Study Clinic }\end{array}$} & \multicolumn{5}{|c|}{ Study Clinic at McCord Hospital } \\
\hline & & $\begin{array}{l}\text { On } \\
\text { Presentation } \\
\text { at Study Clinic }\end{array}$ & $\begin{array}{c}14 \text { Days } \\
\text { after } \\
\text { Presentation }\end{array}$ & $\begin{array}{c}29 \text { Days } \\
\text { after } \\
\text { Presentation }\end{array}$ & $\begin{array}{c}2.5 \mathrm{Mo} \\
\text { after } \\
\text { Presentation }\end{array}$ & $\begin{array}{c}3.5 \mathrm{Mo} \\
\text { after } \\
\text { Presentation }\end{array}$ \\
\hline Rapid screening for $\mathrm{HIV}_{\dagger}^{+}$ & Negative & & & & & \\
\hline EIA & Negative & Positive & & & & \\
\hline CD4+T-cell count (per $\mathrm{mm}^{3}$ ) & & 240 & 357 & 414 & 564 & 443 \\
\hline \multicolumn{7}{|l|}{ Antibodies to HIV antigenst } \\
\hline gpl60 & - & $+/-$ & + & + & + & \\
\hline gpl20 & - & $+/-$ & + & + & + & \\
\hline p65 & - & - & - & - & + & \\
\hline p55 & - & - & + & + & + & \\
\hline p51 & - & - & - & - & + & \\
\hline gp4l & - & - & - & - & + & \\
\hline p40 & - & - & + & + & + & \\
\hline p31 & - & - & - & $+/-$ & $+/-$ & \\
\hline p24 & - & - & + & + & + & \\
\hline pl8 & - & - & $+1-$ & $+/-$ & + & \\
\hline $\begin{array}{l}\text { Interpretation of Western } \\
\text { blot results }\end{array}$ & Negative & Indeterminate & Positive & Positive & Positive & \\
\hline $\begin{array}{l}\text { HIV-1 RNA screening pool } \\
\text { (6 specimens) }\end{array}$ & Positive & & & & & \\
\hline HIV-I RNA copies/ml & 12,445 & $>10$ million & 249,618 & 200,398 & 71,876 & 14,561 \\
\hline \multicolumn{7}{|c|}{$\begin{array}{l}\text { * EIA denotes enzyme immunoassay, and HIV human immunodeficiency virus. } \\
\dagger \text { Two parallel rapid HIV tests were performed on whole blood. Additional HIV tests (two parallel rapid tests, EIA, } \\
\text { Western blot, and HIV-I RNA testing) were performed as part of an acute HIV infection study on a plasma sample } \\
\text { obtained at the first clinic. } \\
7 \text { Antibodies to HIV antigens were determined with the use of Western blot analyses. A minus sign denotes a negative } \\
\text { test result, a plus-minus sign an indeterminate result, and a plus sign a positive result. }\end{array}$} \\
\hline
\end{tabular}

ing tests (four rapid tests and one HIV EIA), a negative HIV-1 Western blot analysis, and detectable HIV RNA from a specimen of blood drawn at the time of her visit to the first clinic (Table 1). This combination of test results is diagnostic of acute HIV infection (Fig. 1). Blood was also obtained from this patient when she presented 13 days later to the McCord Hospital study clinic. HIV testing was performed on this specimen; the HIV EIA was positive, and the supplemental HIV-1 Western blot analysis was indeterminate. Results of an HIV-1 RNA test revealed more than 10 million copies of HIV-1 RNA per milliliter of plasma, which suggest that this patient was in the process of HIV seroconversion (Fig. 1). Two weeks later, the HIV-1 Western blot analysis became positive. When this patient presented to the McCord Hospital clinic, her initial CD4+
T-cell count was 240 cells per cubic millimeter. Although this seems low, it is consistent with the transient decline in the CD4+ lymphocyte count that is characteristic of acute HIV infection ${ }^{13,18}$ (Table 1).

\section{EPIDEMIOLOGY AND PREVENTION OF HIV IN SOUTH AFRICA}

Dr. Quarraisha Abdool Karim: This 19-year-old woman with four lifetime sexual encounters and a 25-year-old male partner has acute HIV infection and an ulcerative sexually transmitted infection. She epitomizes the challenges facing young women in a country where HIV is endemic. With less than $1 \%$ of the world's population, South Africa bears a disproportionate burden of global HIV infections (10\% and 5.5 million people), which 


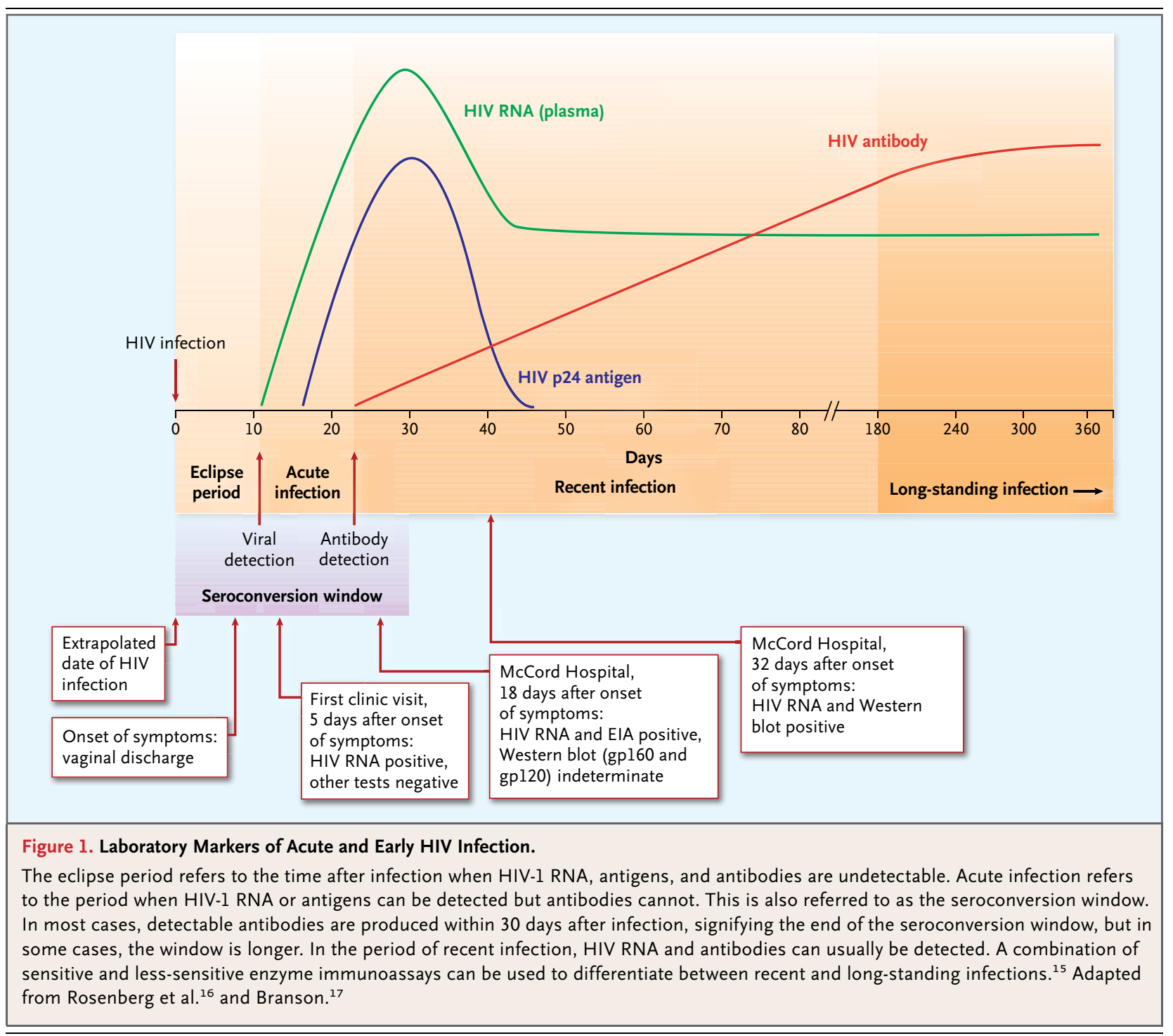

are predominantly sexually transmitted. HIV in South Africa has evolved rapidly - despite a relatively late introduction to the virus - from a prevalence of $0.8 \%$ in the early 1990 s to $26.5 \%$ by 2002 , a percentage that has remained relatively stable. ${ }^{19}$ Although access to antiretroviral therapy is increasing, the rates of new infections remain higher than $5 \%$ per year. ${ }^{20,21}$

Although it may seem surprising that our patient, with a total of only four sexual encounters, has acute HIV infection, her situation is all too common in South Africa. A distinctive characteristic of HIV epidemics in which heterosexual spread is dominant is the difference in infection rates with respect to age and sex, with women acquiring HIV infection at a much earlier age than men. ${ }^{22,23}$ Girls from 15 through 19 years of age have a higher rate of HIV infection, by a factor of 3 to 6 , than their male counterparts, even though first sexual contact occurs at similar ages; nearly peak HIV prevalence in women is reached by the age of 20 years. ${ }^{1,3}$ HIV infection is less common in men before the age of approximately 25 years, the age of this patient's partner. The difference in sexual coupling patterns, in terms of age and sex, and the high prevalence of HIV, continue to drive the southern African epidemic. ${ }^{24}$ In the rural Vulindlela subdistrict of KwaZulu-Natal, the prevalence of HIV infection in pregnant women 19 to 20 years of age, the age of our patient, is $33 \%{ }^{25}$ 


\section{TRANSMISSION}

Although sexual transmission is regarded as the least efficient mode of HIV transmission, this patient illustrates a feature of the South African epidemic: paradoxically high rates of HIV transmission in young women with low coital frequency. Although we do not have information about the patient's partner, the higher the partner's viral load, the greater the probability of male-to-female and female-to-male HIV transmission. ${ }^{26}$ During the asymptomatic period of infection, the viral load is lowest and the risk of transmission is lowest. However, in the presence of sexually transmitted infections, the viral load during the asymptomatic period can reach elevated levels similar to those seen during acute symptomatic HIV infection, and the risk of HIV transmission is elevated, especially if a genital ulcer is present. This patient and possibly her partner had an ulcerative sexually transmitted infection that probably facilitated transmission of the virus.

\section{PREVENTING HIV INFECTION}

This case presents us with a key question: What can be done to prevent infection with HIV or other sexually transmitted pathogens? In the three decades of the HIV pandemic, substantial advances have been made in treating the acquired immunodeficiency syndrome (AIDS) and preventing transmission of HIV from infected mothers to infants. In contrast, preventing sexual transmission, which accounts for more than $80 \%$ of all HIV infections, remains a challenge. A systematic review of 37 randomized, controlled trials involving HIV prevention has revealed that only 5 showed successful HIV prevention. ${ }^{27}$

The so-called ABC approach (abstain, be faithful, and use condoms) remains the foundation of HIV prevention. Additional approaches to prevention include male circumcision, as well as counseling and HIV testing to provide knowledge of HIV status. Unfortunately for this young woman, her partner was unwilling to use a condom. For sexually active women who are unable to negotiate monogamy or condom use with their sexual partner, there are currently no options for the prevention of HIV. This lack of options underscores the urgent need for protective methods that can be initiated by women, such as effective microbicides or the prophylactic use of antiretroviral drugs for the prevention of infection. ${ }^{28}$

The Centre for the AIDS Programme of Re- search in South Africa (CAPRISA) recently announced the results from the CAPRISA 004 study, a phase $2 \mathrm{~B}$, randomized, controlled trial of $1 \%$ tenofovir gel that showed a 39\% reduction in HIV acquisition in women ${ }^{29}$ and a $51 \%$ reduction in the acquisition of herpes simplex virus type 2.25 Although it is too late for prophylactic therapy for our patient, this therapy provides hope that HIV prevention methods that can be initiated by women are within our grasp.

\section{ALTERING THE TRAJECTORY OF THE HIV EPIDEMIC IN SOUTHERN AFRICA}

What can we do for patients, and how can we intervene to reduce further transmission of HIV? In addition to treating the sexually transmitted infections and reducing the viral load for both personal and public benefit, counseling and support to reduce the risk of HIV is critical. Although interventions regarding prevention have consistently shown a significant effect, ${ }^{30}$ these efforts in care and treatment programs unfortunately remain small or nonexistent.

A critical challenge in altering the trajectory of the HIV epidemic in southern Africa is the poor recognition of personal risk, as reflected in low rates of HIV testing. This patient was aware of HIV and its sexual transmission but remained at risk for acquiring HIV infection. The South African government recently launched a substantive campaign to promote knowledge of HIV status and a program for medical male circumcision. The South African antiretroviral treatment program is the largest in the world, but coverage remains at about $30 \%$ and a lot more needs to be done to reach the target of $80 \%$. Clinics for the care and treatment of patients with AIDS need to expand their services to include counseling regarding reducing the risk of HIV, as well as reproductive health services to suppress the transmission of HIV.

DISCUSSION OF MANAGEMENT

Dr. Rajesh Gandhi (Medicine): Dr. Venter, could you tell us how you would treat this patient?

Dr. Venter: After a diagnosis of acute HIV infection has been established, management priorities should include aggressive retention of patients in care programs, treatment and follow-up of the vaginal discharge and ulcer, tuberculosis prophylaxis, fertility advice, timely initiation of anti- 
retroviral medication, and appropriate interventions to prevent further transmission.

The genital ulcer and vaginal discharge should respond well to therapy according to the new South African guidelines, which - mindful of the rise in local fluoroquinolone-resistant gonorrhea, as well as the increase in herpes as a cause of ulcers - now advocate the use of third-generation cephalosporins in place of ciprofloxacin for uncomplicated vaginal discharge (with metronidazole and doxycycline) and acyclovir for the treatment of ulcers (with erythromycin and penicillin). ${ }^{5,12}$ If the symptoms do not resolve, a more focused investigation should be performed after it is determined whether the patient adhered to her treatment regimen. Treatment of the sexually transmitted disease may decrease the plasma viral load, which may slow the progression to AIDS. ${ }^{31}$

Treatment of the patient's recently acquired HIV infection is not so easy. It is clear that a large amount of immunologic damage is wrought during the first few months after infection. ${ }^{32}$ However, antiretroviral treatment of acute HIV infection is controversial, with no firm evidence of clinical benefit, although at least one study suggests immunologic benefit. ${ }^{33}$ In fact, the timing of the initiation of antiretroviral therapy in persons with HIV is still under debate, and we are awaiting evidence from randomized, controlled trials showing the benefits of treatment at higher CD4+ T-cell counts ( $>350$ cells per cubic millimeter). ${ }^{34}$ Currently, guidelines for most developed countries advocate treating asymptomatic HIV infection in patients who have a CD4+ T-cell count below 500 cells per cubic millimeter, with many suggesting treatment at higher CD4+ T-cell counts. ${ }^{35}$ World Health Organization guidelines take a more conservative approach, suggesting treatment at the threshold CD4+ T-cell count of 350 cells per cubic millimeter for asymptomatic patients unless they are pregnant. ${ }^{36}$ South African guidelines recommend treatment for healthy patients with HIV infection who are not pregnant and have a CD4+ count of 200 cells or less per cubic millimeter, due to the perceived costs and burden on available services. ${ }^{37}$ Our patient would not receive antiretroviral treatment until her CD4+ count falls to 200 cells or less per cubic millimeter, she becomes pregnant, or an AIDS-defining illness develops.

One of the thorniest problems in HIV management is keeping patients in a care program when they are not provided antiretroviral treatment. The retention rate is very poor. In South Africa's program, the average age at the initiation of antiretroviral treatment in women is the early $30 \mathrm{~s}$, and usually the CD4+ count is below 100 cells per cubic millimeter. ${ }^{38}$ Retaining this teenage woman in care is going to be a major challenge. Before the patient leaves the clinic, the attending clinician should carefully explain to her the immediate and long-term care plan and the importance of consistent follow-up.

Isoniazid should be started for tuberculosis prophylaxis, after active tuberculosis is ruled out through basic screening for symptoms, with careful repeat screening for symptoms. South African guidelines have removed the need for a purified protein derivative skin test. In anticipation of her wanting children, she should be given advice about fertility and contraception, advised against pregnancy while her viral load is likely to be high, and encouraged to get informed health care advice regarding the optimal timing of a pregnancy. Any suspicion of pregnancy should be confirmed immediately, so that treatment to prevent motherto-child transmission can be initiated as soon as possible.

Finally, the patient requires intensive counseling regarding safe-sex practices. She reports having had only one sex partner. If this is true, the partner is HIV-positive; if it is not true, there is a high possibility of concurrent partners becoming infected, since acute HIV infection is associated with very high viral loads and transmission risk. ${ }^{39}$ Her partner should be encouraged to receive HIV counseling and testing. If issues such as gender violence preclude discussion with the partner, barrier contraception or abstinence are alternative strategies to be considered.

Dr. Gandhi: Dr. Ndung'u, would you tell us what happened to this patient?

Dr. Ndung'u: The patient is currently seen in the clinic every 6 months. At the time of her last visit, 1 year after the onset of her illness, her CD4+ T-cell count was 514 cells per cubic millimeter and her plasma HIV-1 RNA load was 30,644 copies per milliliter. She remains off antiretroviral therapy. During one visit, she reported a sore throat, headache, and lymphadenopathy. She is otherwise well.

$$
\text { FINAL DIAGNOSIS }
$$

Acute HIV infection with concurrent sexually transmitted infection. 
This case was presented at the Fourth Annual Workshop on Advanced Clinical Care - AIDS in Durban, South Africa, September 30-October 1, 2010. The workshop was sponsored by the Harvard University Center for AIDS Research, McCord Hospital (Durban), the University of KwaZulu-Natal, the South African HIV Clinicians Society, and the Department of Health of KwaZulu-Natal.

No potential conflict of interest relevant to this article was reported.
Disclosure forms provided by the authors are available with the full text of this article at NEJM.org.

We thank Vivek Naranbhai for assistance with the preparation of slides and presentation of the epidemiology section at the conference, and Drs. Henry Sunpath, Mahomed-Yunus S. Moosa, and Rajesh Gandhi for organizing the workshop.
REFERENCES

1. Pettifor AE, Rees HV, Kleinschmidt I, et al. Young people's sexual health in South Africa: HIV prevalence and sexual behaviors from a nationally representative household survey. AIDS 2005;19:1525-34 2. Pettifor AE, Hudgens MG, Levandowski BA, Rees HV, Cohen MS. Highly efficient HIV transmission to young women in South Africa. AIDS 2007;21:861-5.

3. Shisana O, Rehle T, Simbayi LC, Zuma K, Jooste S, Pillay-van-Wyk V. South African national HIV prevalence, incidence, behaviour and communication survey 2008 : A turning tide among teenagers? Cape Town, South Africa: HSRC Press, 2009.

4. O'Farrell MJ, Studier EH, Ewing WG. Energy utilization and water requirement of captive Myotis thysanodes and Myotis lucifugus (Chiroptera). Comp Biochem Physiol A Comp Physiol 1971;39:549-52.

5. Lewis DA, Maruma E. Revision of the national guideline for first-line comprehensive management and control of sexually transmitted infections: what's new and why? South Afr J Epidemiol Infect 2009;24:6-9.

6. Lewis DA, Venter JME, Mhlongo S, Müller E, Radebe F. Think herpes, think HIV: results of aetiological surveillance among genital ulcer patients in South Africa 2006-2008. In: Programme and abstract book of the 18th ISSTDR in conjunction with BASHH Congress. London International Society for Sexually Transmitted Diseases Research, 2009. abstract. 7. O'Farrell N, Morison L, Moodley P, et al. Genital ulcers and concomitant complaints in men attending a sexually transmitted infections clinic: implications for sexually transmitted infections management. Sex Transm Dis 2008;35:545-9.

8. Kahn JO, Walker BD. Acute human immunodeficiency virus type 1 infection. N Engl J Med 1998;339:33-9.

9. Mhlongo S, Magooa P, Müller EE, et al. Etiology and STI/HIV coinfections among patients with urethral and vaginal discharge syndromes in South Africa. Sex Transm Dis 2010;37:566-70.

10. First line comprehensive management and control of sexually transmitted infections (STIs): protocol for the management of a person with a sexually transmitted infection according to the Essential Drug List. Pretoria, South Africa: Department of Health, 2008:1-18.

11. O'Farrell N, Hoosen AA, Coetzee KD, van den Ende J. Genital ulcer disease: accuracy of clinical diagnosis and strategies to improve control in Durban, South Africa. Genitourin Med 1994;70:7-11.

12. Vanhems P, Dassa C, Lambert J, et al. Comprehensive classification of symptoms and signs reported among $218 \mathrm{pa}-$ tients with acute HIV-1 infection. J Acquir Immune Defic Syndr 1999;21:99-106.

13. Case Records of the Massachusetts General Hospital (Case 11-2009). N Engl J Med 2009;360:1540-8.

14. Guidance on provider-initiated HIV testing and counselling in health facilities. Geneva: World Health Organization, 2007. (http://whqlibdoc.who.int/publications/ 2007/9789241595568_eng.pdf.)

15. Janssen RS, Satten GA, Stramer SL, et al. New testing strategy to detect early HIV-1 infection for use in incidence estimates and for clinical and prevention purposes. JAMA 1998;280:42-8. [Erratum, JAMA 1999;281:1893.]

16. Rosenberg ES, Brennan CA, Claessens $\mathrm{C}$, et al. Criteria for laboratory testing and diagnosis of human immunodeficiency virus infection: approved guideline. (CLSI document M53-A.) Wayne, PA: Clinical Laboratory Standards Institute (in press). (http://www.CLSI.org.)

17. Branson BM. The future of HIV testing. J Acquir Immune Defic Syndr 2010; 55:Suppl 2:S102-S105.

18. Kassutto S, Maghsoudi K, Johnston $\mathrm{MN}$, et al. Longitudinal analysis of clinical markers following antiretroviral therapy initiated during acute or early HIV type 1 infection. Clin Infect Dis 2006;42: 1024-31.

19. National HIV and syphilis antenatal seroprevalence survey in South Africa: 2009. Pretoria, South Africa: National Department of Health, 2009.

20. Bärnighausen T, Tanser F, Gqwede Z, Mbizana C, Herbst K, Newell ML. High HIV incidence in a community with high HIV prevalence in rural South Africa: findings from a prospective populationbased study. AIDS 2008;22:139-44.

21. Gouws E. HIV Incidence rates in South Africa. In: Abdool Karim S, Abdool Karim Q, eds. HIV/AIDS in South Africa. Cape Town, South Africa: Cambridge University Press, 2010:74-84.

22. Abdool Karim Q. Heterosexual transmission of HIV - the importance of a gendered perspective in HIV prevention.
In: Abdool Karim SS, Abdool Karim Q, eds. HIV/AIDS in South Africa. 2nd ed. Cape Town, South Africa: Cambridge University Press, 2010:285-304.

23. Gregson S, Nyamukapa CA, Garnett GP, et al. Sexual mixing patterns and sexdifferentials in teenage exposure to HIV infection in rural Zimbabwe. Lancet 2002; 359:1896-903. [Erratum, Lancet 2002;360 1102.]

24. Abdool Karim SS, Churchyard GJ, Abdool Karim Q, Lawn SD. HIV infection and tuberculosis in South Africa: an urgent need to escalate the public health response. Lancet 2009;374:921-33.

25. Abdool Karim Q, Abdool Karim SS CAPRISA 004: effectiveness \& safety of vaginal microbicide $1 \%$ tenofovir gel for prevention of HIV infection in women. Presented at the XVIII International AIDS Conference 2010, Vienna, Austria, July 18-23, 2010.

26. Quinn TC, Wawer MJ, Sewankambo $\mathrm{N}$, et al. Viral load and heterosexual transmission of human immunodeficiency virus type 1. N Engl J Med 2000;342:921-9. 27. Padian NS, McCoy SI, Balkus JE, Wasserheit JN. Weighing the gold in the gold standard: challenges in HIV prevention research. AIDS 2010;24:621-35.

28. Stein ZA. HIV prevention: the need for methods women can use. Am J Public Health 1990;80:460-2.

29. Abdool Karim Q, Abdool Karim SS, Frohlich JA, et al. Effectiveness and safety of tenofovir gel, an antiretroviral microbicide, for the prevention of HIV infection in women. Science 2010;329:1168-74.

30. Crepaz N, Lyles CM, Wolitski RJ, et al. Do prevention interventions reduce HIV risk behaviours among people living with HIV? A meta-analytic review of controlled trials. AIDS 2006;20:143-57.

31. Modjarrad K, Vermund SH. Effect of treating co-infections on HIV-1 viral load: a systematic review. Lancet Infect Dis 2010;10:455-63.

32. Brenchley JM, Douek DC. The mucosal barrier and immune activation in HIV pathogenesis. Curr Opin HIV AIDS 2008;3: 356-61.

33. Moir S, Buckner CM, Ho J, et al. $B$ cells in early and chronic HIV infection: evidence for preservation of immune function associated with early initiation of antiretroviral therapy. Blood 2010;116: 5571-9. 
34. Siegfried N, Uthman OA, Rutherford GW. Optimal time for initiation of antiretroviral therapy in asymptomatic, HIVinfected, treatment-naive adults. Cochrane Database Syst Rev 2010;3:CD008272.

35. Panel on Antiretroviral Guidelines for Adults and Adolescents. Guidelines for the use of antiretroviral agents in HIV-1-infected adults and adolescents. Washington, DC: Department of Health and Human Services, December 1, 2009: 1-161.

36. Rapid advice: antiretroviral therapy for HIV infection in adults and adolescents. Geneva: World Health Organization, November 2009.

37. Clinical guidelines for the management of HIV \& AIDS in adults and adolescents. Pretoria, South Africa: National Department of Health, 2010.
38. Boulle A, Bock P, Osler M, et al. Antiretroviral therapy and early mortality in South Africa. Bull World Health Organ 2008;86:678-87.

39. Pilcher CD, Tien HC, Eron JJ Jr, et al. Brief but efficient: acute HIV infection and the sexual transmission of HIV. J Infect Dis 2004;189:1785-92.

Copyright $\odot 2011$ Massachusetts Medical Society.

LANTERN SLIDES UPDATED: COMPLETE POWERPOINT SLIDE SETS FROM THE CLINICOPATHOLOGICAL CONFERENCES Any reader of the Journal who uses the Case Records of the Massachusetts General Hospital as a teaching exercise or reference material is now eligible to receive a complete set of PowerPoint slides, including digital images, with identifying legends, shown at the live Clinicopathological Conference (CPC) that is the basis of the Case Record. This slide set contains all of the images from the CPC, not only those published in the Journal. Radiographic, neurologic, and cardiac studies, gross specimens, and photomicrographs, as well as unpublished text slides, tables, and diagrams, are included. Every year 40 sets are produced, averaging 50-60 slides per set. Each set is supplied on a compact disc and is mailed to coincide with the publication of the Case Record.

The cost of an annual subscription is $\$ 600$, or individual sets may be purchased for $\$ 50$ each. Application forms for the current subscription year, which began in January, may be obtained from the Lantern Slides Service, Department of Pathology, Massachusetts General Hospital, Boston, MA 02114 (telephone 617-726-2974) or e-mail Pathphotoslides@partners.org. 